\title{
Towards an Open Learning Infrastructure for Open Educational Resources: Abundance as a Platform for Innovation
}

\author{
Erik Duval, Katrien Verbert, and Joris Klerkx \\ Dept. Computerwetenschappen, K.U.Leuven, B3000 Leuven, Belgium \\ \{Erik.Duval, Katrien.Verbert, Joris.Klerkx\}@cs.kuleuven. be
}

\begin{abstract}
This paper explains how we have contributed to the development of an open learning infrastructure that manages and makes available Open Educational Resources. By removing friction between people and resources, we can leverage the long tail of learning resources, so that the abundance of learning resources will act as a platform for innovation.
\end{abstract}

Keywords: open educational resources, learning technology standards.

\section{Introduction}

This paper briefly presents some of the main outcomes of our work on learning objects, metadata and interoperability. The basic idea is that we are beginning to realize our early vision of an open learning infrastructure that enables scaling up technical facilities for Technology Enhanced Learning (TEL) to a global scale $[22]$.

Our focus for the last 15 years has been on providing easy access to all learning resources, for all teachers and for all students [10]. In a life-long learning context: this means for all of us. Obviously, there are some basic challenges in terms of internet connectivity, but as access to the network proliferates, so will access to digital learning resources. Our vision is very similar to what Hermann Maurer presented in [14].

Making more content and other resources more easily available will not solve all the problems related to education, training and learning in general. However, we do believe that the abundance thus created will act as a motor for innovation, much in the same way that ubiquitous access to music has profoundly changed the way consumers interact with music and, as a consequence, the music industry.

Indeed, there is an assumption underlying much of our work that, at some point, more is not just more, but creates a qualitative tipping point as well. One of the reasons why we pursue global access to all learning resources for everyone is because we believe that there will be a long tail effect [7] of learning material: basically, there may be few students and teachers of more specialized topics like Egyptian hieroglyphs; but on a global scale, there are sufficient people interested in this topic from a learning perspective to create a sustainable community for share and reuse.

C.S. Calude, G. Rozenberg, A. Salomaa (Eds.): Maurer Festschrift, LNCS 6570, pp. 144-156, 2011. (C) Springer-Verlag Berlin Heidelberg 2011 
In fact, abundance not only creates new opportunities, but can also create new problems, such as the Paradox of choice [18] that arises when the cognitive and emotional overhead of dealing with abundance overshadows the benefits of a larger number of options. We consider such problems as drivers for innovation, as they encourage us to research novel ways to enable interested parties to find or be alerted about relevant resources in appropriate ways [15].

Since almost 15 years now, we pursue this goal of share and reuse in the ARIADNE Foundation that has developed a network of repositories of learning materials, with associated tools for harvesting and search [2]. More recently, we created a world-wide alliance of similar organizations, a network of networks of repositories: Globe [3] that interconnects about a dozen such networks, using the ARIADNE technology as its technical backbone infrastructure.

This paper is structured as follows: section 2 presents how we are evolving towards an open learning infrastructure. Section 3 illustrates how the resulting abundance is beginning to act as a motor for innovation. Section 4 presents some ongoing and future work. We then present our conclusion and list bibliographical references.

\section{Background: Towards an Open Learning Infrastructure}

\subsection{Introduction}

In order to realize an open learning infrastructure, we have contributed to the development of a number of open standards, that enable the interconnection between different infrastructures, or even the development of an integrated infrastructure from heterogeneous independently developed building blocks [13].

The main relevant standards include:

- IEEE LTSC Learning Object Metadata (LOM) [5]: a hierarchical structure of some 70 metadata elements that can be used to describe learning resources;

- CEN WSLT Simple Query Interface (SQI) [6]: a conceptual protocol for searching in repositories with LOM descriptions;

- CEN WSLT Simple Publishing Interface (SPI) [21]: a language for ingesting new metadata and/or resources into a learning object repository;

- Open Archives Initiative Protocol for Metadata Harvesting (OAI-PMH) [23]: a protocol for periodically retrieving all updated or new metadata from a repository.

Most of these standards are conceptual: this means that they are abstract and can be bound to a specific representation, for instance as a SOAP or REST interface for the interfaces or protocols, or as JSON or XML for LOM. The main idea is that they would thus be more resilient to change of technology bindings, an important aspect as the standards are intended to remain relevant for a decade or longer. 


\subsection{The ARIADNE Infrastructure}

The main design principle in ARIADNE is to make everything disappear but the benefits by removing friction when one wants to share and reuse learning resources. For that reason, we have built an infrastructure to make this happen. Figure 1 illustrates the ARIADNE infrastructure.
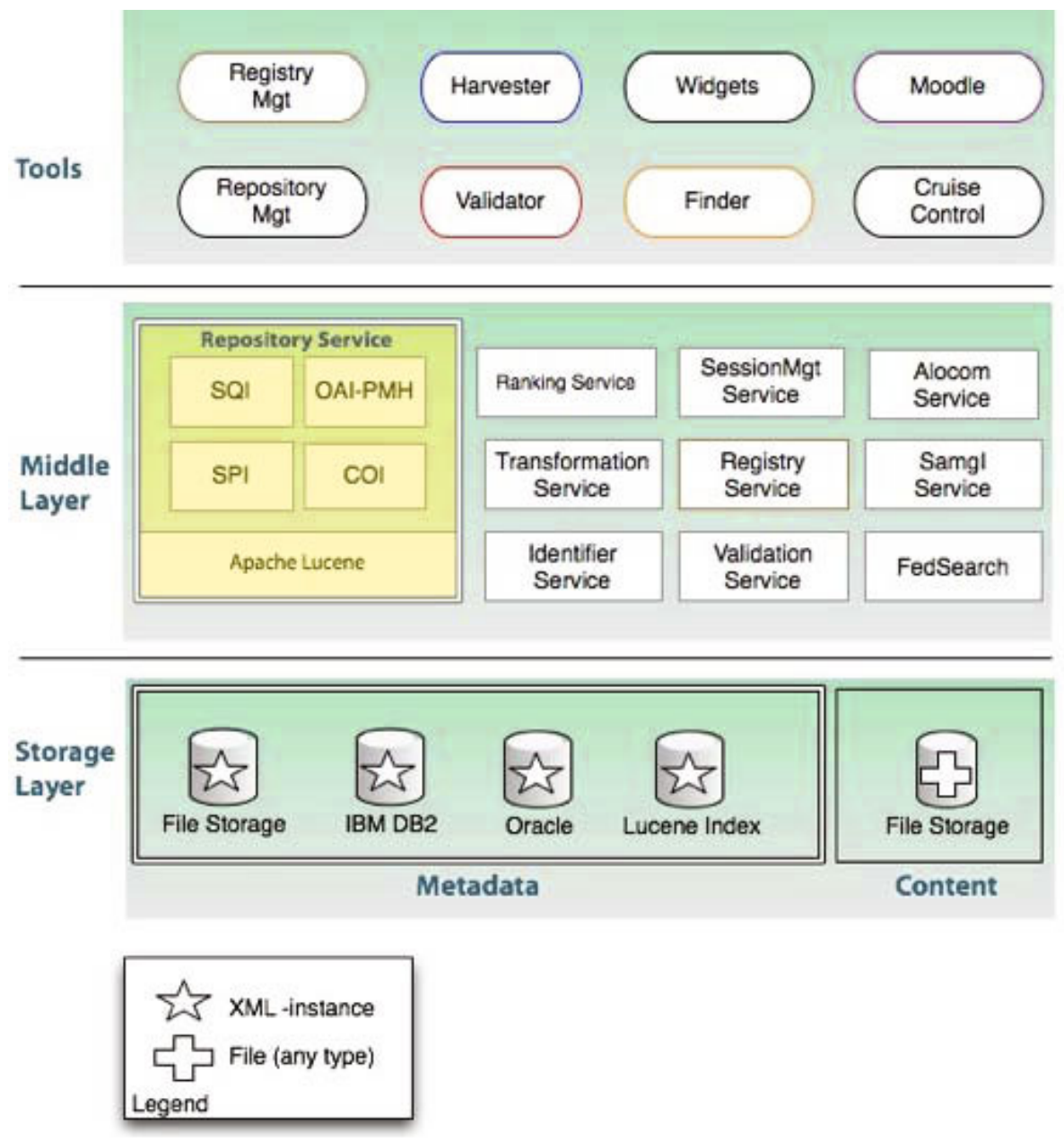

Fig. 1. The layered ARIADNE infrastructure

Basically, the infrastructure is composed of three layers:

1. The Tool layer hides protocols and standards for end users in a toolset that provides access to learning material through web applications, information visualization, mobile information devices, multi-touch displays and mash-up applications (see also below). A more administration oriented application is 
the ARIADNE harvester that is able to harvest metadata with OAI-PMH from a content provider into the ARIADNE storage layer.

2. The Middle Layer offers persistent management of learning objects and metadata through a set of services, such as:

- the repository services that allow for the management of learning objects, relying on standards such as IEEE LTSC LOM [5], CEN WSLT SQI [6], CEN WSLT SPI [21] and OAI-PMH [23];

- the registry services that manage information on learning object repositories, including the information necessary for other infrastructure components to select protocols supported by a given repository;

- an identifier service for providing unique identifiers for learning objects managed in the infrastructure,

- a validation service that checks both the syntactic and semantic validity of metadata instances against multiple standards, specifications and their application profiles,

- a transformation service that converts metadata from one format, for instance Dublin Core, into another format, for instance the ARIADNE LOM application profile.

3. The Storage Layer allows for storing both content and metadata in diverse repositories depending on the choice of the administrator who deploys the ARIADNE infrastructure.

Detailed information about these services has been published in [13]. In the following section, we show how these services and tools can be orchestrated to add new repositories to the ARIADNE network.

\subsection{Adding New Repositories}

Figure 2 illustrates how ARIADNE services and tools can be orchestrated to connect the repositories of content providers X (left side of figure) and Y (upper right side) with the ARIADNE network. As an example, we explain how we recently integrated the JorumOpen repository [19] in the ARIADNE network. All resources from JorumOpen are described in the Dublin Core (DC) metadata schema. JorumOpen had already implemented an OAI-PMH target on top of the repository. To connect to ARIADNE, this OAI-PMH target is registered into the ARIADNE Registry (step 1a) where administrative and technical information is added about the repository, such as the title, description, contact person, supported protocols used in his repository (OAI-PMH and DC metadata), etc. The registry uses an RSS feed to alert all (step 1.2) of its client tools about the new repository that has been added.

In response to this alert, the ARIADNE harvester tool issues a query (step 2) to the registry to obtain all relevant information about this repository. In step 3 , the harvester performs the following steps:

- The Dublin Core metadata is harvested from JorumOpen using the OAIPMH protocol (step 3.1). 


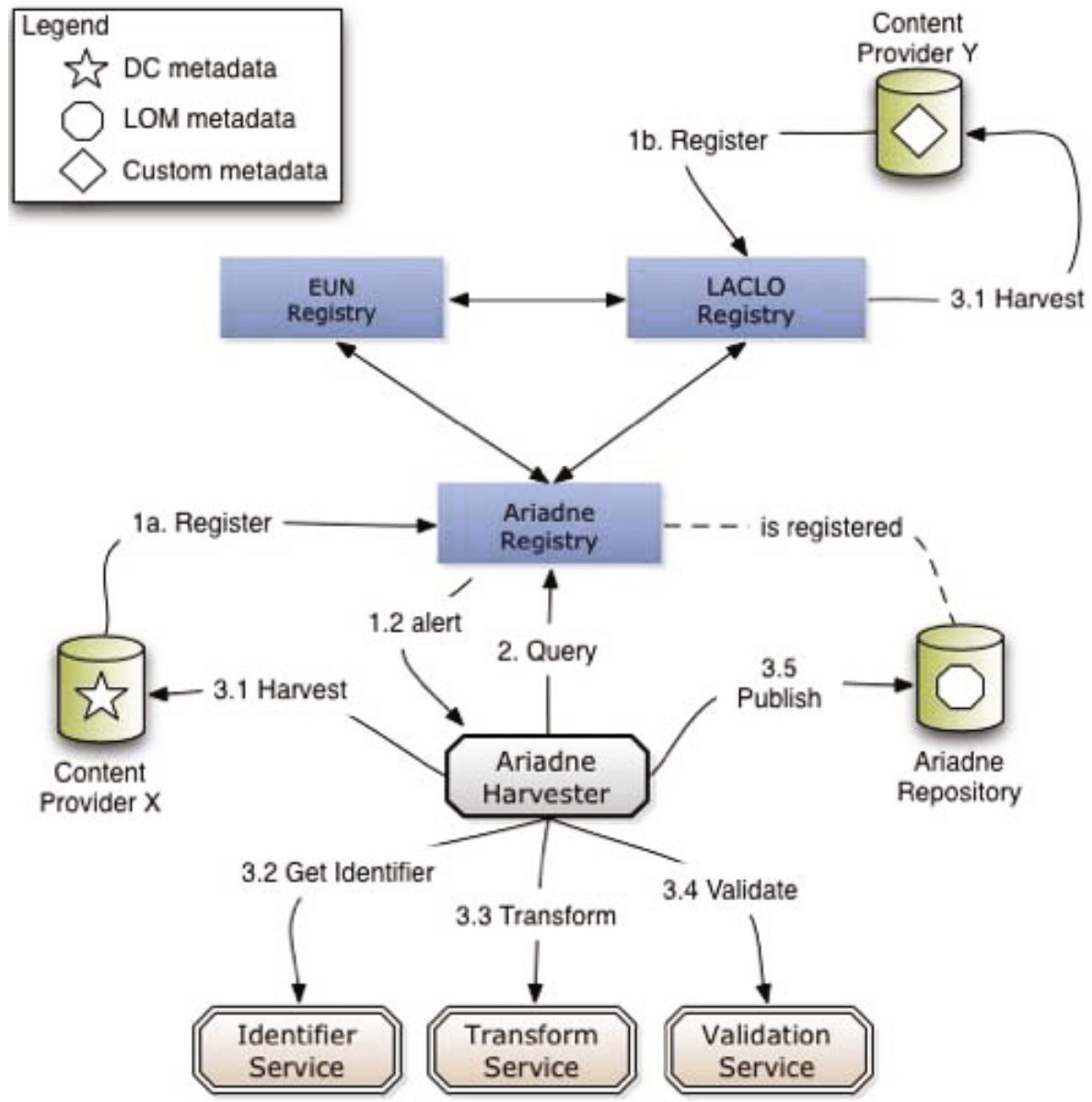

Fig. 2. Adding new repositories

- From experience in various projects, we have learned that we cannot rely on the identifiers that are added by content providers because duplicates may arise. That is why the harvester calls the Identifier service (step 3.2) to generate a unique identifier, which is added to the metadata instances of provider JorumOpen.

- A call to the transform service takes care of the conversion from Dublin Core to the ARIADNE LOM Application Profile (step 3.3).

- Faulty metadata instances often result in errors or inconsistencies in tools and user interfaces. All metadata instances are therefore validated against the validation scheme (step 3.4), and invalid instances are discarded. Mapping metadata from a local metadata scheme to a global one typically happens either by manually editing one record at the time, or in batch with a script written specifically for this mapping. Our experience has shown that both of these methods make this a very error-prone process. For example, 
in the eContentplus project MELT, $90 \%$ of the original metadata from content providers resulted in validation errors. The reports of the ARIADNE validation service helped the content providers to dramatically decrease this number to less than $5 \%$.

- The harvester uses the Simple Publishing Interface (SPI) specification to publish the harvested and validated metadata into the ARIADNE target that is registered as well in the registry. All content from JorumOpen can thus be found in ARIADNE (step 3.5) after the harvester has completed the cycle from steps 3.1 to 3.5 .

It is very important to emphasize the semi-automated nature of this process: all that is required is the registration of some administrative and technical data in the registry. From there on, there may be alerts for manual intervention if metadata does not validate or if the services are not available, but otherwise the process proceeds automatically. This is very important if we want to make this infrastructure scale world-wide.

\subsection{From ARIADNE to the Rest of the World}

Through various projects over the years, the ARIADNE infrastructure has proven to be capable of providing flexible, effective and efficient access to largescale collections of learning resources. At the time of writing, the ARIADNE registry contains information about 79 repositories that enables access to more than a million learning resources.

In fact, the ARIADNE resources are also available outside of the strict context of the ARIADNE network itself. The registry is interoperable with third-party registries, so that all information added in one registry is automatically synchronized to the other registries. In this way, content that is collected by ARIADNE can automatically be found in other networks as well. This also works the other way around. If another content provider $\mathrm{Y}$ is added to the LACLO registry [4], this information is synchronized with the ARIADNE registry. Cycle 3.1 to 3.5 described in the previous section then starts all over again, such that those resources become available for ARIADNE users as well.

All harvested metadata is accessible and open to the world:

1. First of all, the complete LOM/XML dataset can be harvested through the OAI-PMH protocol.

2. Secondly, the LOM/XML dataset can be queried through a SOAP binding that implements the Simple Query Interface (SQI) that supports many types of search technologies.

3. Thirdly, a REST API with a JSON binding of LOM allows you to specify queries and aspects of the result format (like resultListSize, resultformat, languages, etc.). Results can be returned in both XML and JSON result formats.

4. Finally, and a bit more experimental in nature, we have also made available the same data as a SPARQL target on top of an RDF binding of LOM. 


\subsection{Search and Find}

The ARIADNE infrastructure has enabled us to collect more than a million learning resources. To enable efficient and fast search on top of this collection, we make use of the open source Apache Lucene and SOLR frameworks that provide powerful, accurate, efficient and facetted search algorithms.

Because search performance is really essential for a satisfactory user experience, we frequently run a series of benchmarking tests on top of our search services. At the time of writing, we achieve an average $15 \mathrm{~ms}$ response time during stress testing our services. These stress tests are performed with the help of Apache Jmeter, a Java desktop application designed to load test functional behavior and measure performance of web applications. Although these results are very acceptable, we are currently investigating multiple alternatives for further improving these results when we scale up the number of resources in our network. Options we are currently exploring include:

- In-memory loading of the complete search index,

- Reducing index size by only including a subset of the metadata elements,

- Distributed search over multiple servers.

The ARIADNEFinder is our state-of-the-art web tool for querying: the screenshot in figure 3 shows how we deployed this in the context of GLOBE. The tool enables the end user to issue a keyword search in the top middle part of the interface. The left pane supports facetted search on the results, by selecting

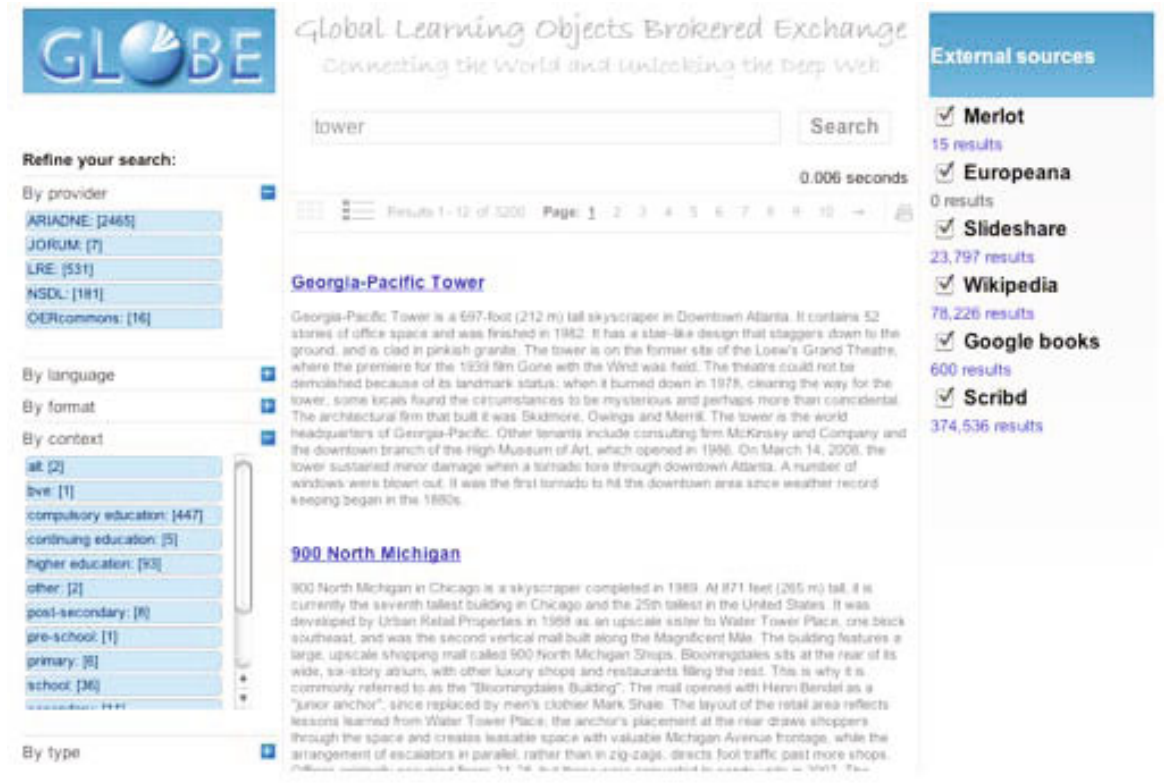

Fig. 3. ARIADNE Finder deployed for GLOBE 
facets and values to include relevant results or to exclude results that are of less importance. On the right pane, one can simultaneously issue a query to external sources of material. Typically, for those targets, it does not make sense to harvest all of their resources. In the wikipedia case for example, the resources are very volatile so instead of harvesting those resources every day, we prefer to use their open search API to issue queries to them and federate the results back into the overall search interface.

\section{Abundance as a Platform for Innovation}

\subsection{Introduction}

As we mentioned in the introduction, an open learning infrastructure will replace the older problem of scarcity of learning resources by a situation of abundance, so that more effective and efficient ways to make use of that abundance will be required.

We see at least three ways to achieve this goal:

1. integration: rather than providing an external, extra tool for teachers or learners to find relevant resources, we can integrate with their mainstream authoring or teaching or learning workflow and suggest relevant material when appropriate (section 3.2);

2. visualization: in order to provide more sophisticated interaction with learning resources, rich visualizations can be developed where learners and teachers navigate an information space, potentially making use of novel interaction techniques provided by multi-touch large scale displays (section 3.3);

3. analytics: through a careful analysis of detailed tracking of what teachers and learners do, we can obtain a clearer idea of what the user is trying to do and how well he is achieving his goal, which opens up opportunities for advanced user support (section 3.4).

No doubt, there will be other approaches that leverage the emergent abundance in other ways...

\subsection{Integration}

As mentioned above, teachers and learners can leverage the abundance of learning content in much more flexible ways if we can integrate access to these resources in more subtle ways in their existing workflows, rather than sending them to a dedicated web site or tool.

A successful example of this approach is the component that we have integrated into the Moodle Learning Management System (LMS) [9]. As illustrated by figure 4, when users want to upload a document in their course, our software enables them to search in ARIADNE from within Moodle. In addition to removing friction from the user experience, this also provides us with opportunities to enrich the typical keyword search from the teacher with background knowledge about the course he is working on, including the topic, the student target audience, the technical requirements, etc. 
Moodle test course.
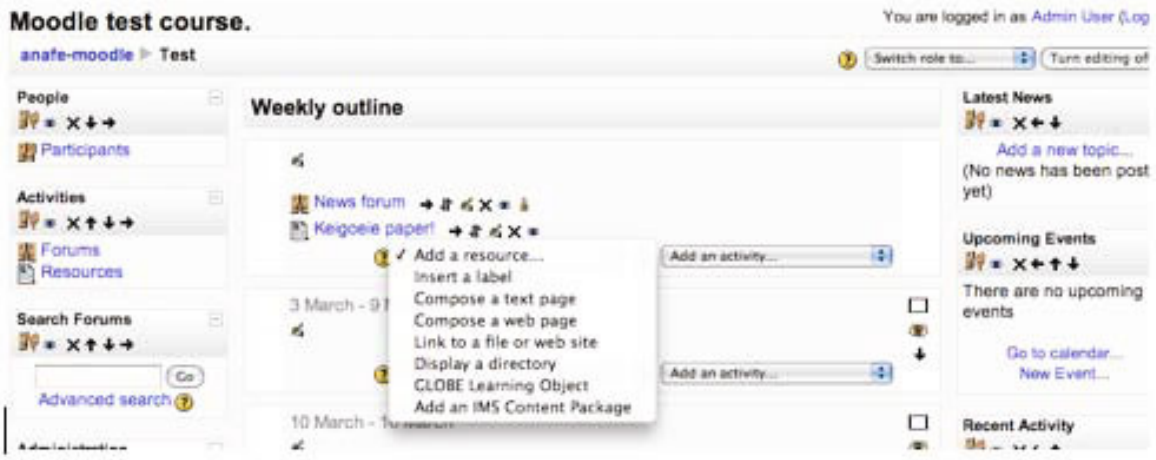

Fig. 4. Searching ARIADNE from within Moodle

\section{Moodle test course.}

You are logoed in as Admin Uner Rog

anafe-moodle > Test $>$ Resources > Edating Resource

\section{ZAdding a new Resource}

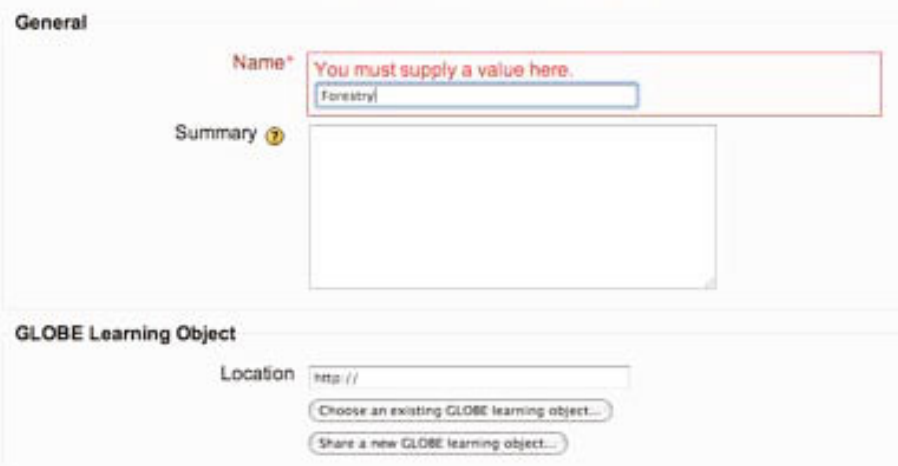

Fig. 5. Inserting resources in Moodle and ARIADNE

Similarly, we also enable a teacher to upload a new resource not only in a Moodle course, but also in the ARIADNE repository, without any additional effort or without leaving the familiar learning environment. This is illustrated in figure 5. In this case, we can make use of the contextual information that the LMS provides to enrich the automatic generation of metadata that accompany the resource in the repository.

We have developed similar interfaces from within the Blackboard learning environment, Microsoft Powerpoint and the LAMS authoring tool. A widget for integration into a Personal Learning Environment (PLE) is under development.

\subsection{Visualization}

When teachers and learners do want to interact explicitly with the abundance of resources available to them, we can use information visualization techniques to provide them with a rich environment for exploration and discussion. 


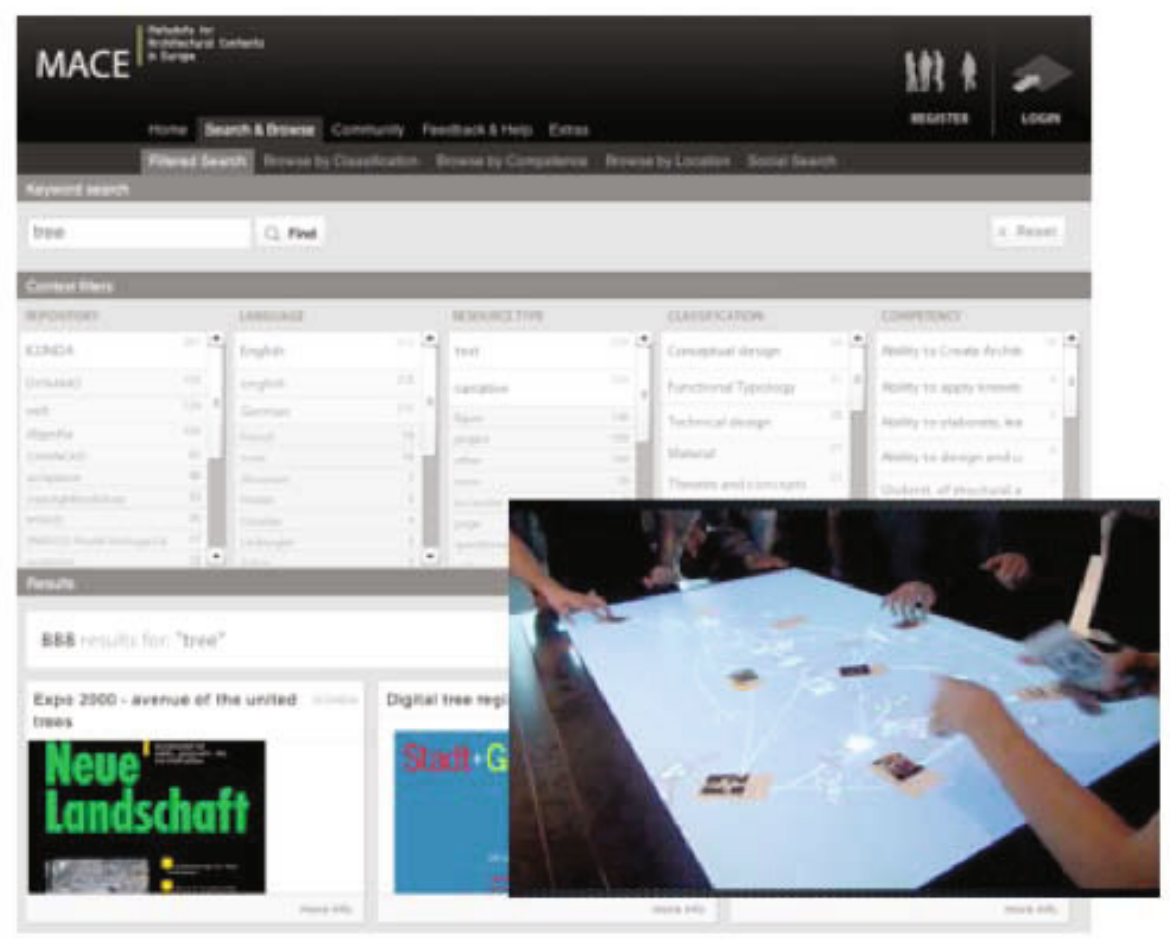

Fig. 6. The Maeve table

A successful example of this approach is the maeve table that provides a large reactable for exploring learning resources about architects, architectural styles, building techniques, etc. [16] - see figure 6. Learners and teachers can manipulate cards that represent the information and put them on an interactive surface to display associated concepts and relations to other information. In this way, learners and teachers can explore a rich information space.

An alternative, more conventional access paradigm is the web portal, shown in figure 6, which uses information visualization techniques such as elastic lists [20], that allows for browsing multi-facetted data structures.

\subsection{Analytics}

In recent years, researchers are focusing increasingly on the need for better measurement, tracking, analysis and visualization of data about learners. Research on learning analytics [1] evolved that describes the set of activities to help understand and optimize learning and the environments in which it occurs.

The capturing of user activities is often researched as a basis to gather and analyze behavior of learners [11][8]. Several models have been elaborated to track user interactions with tools and resources, such as read and write actions on documents or even keystrokes. Examples of models that are used in a Technology Enhanced Learning context are the Contextualized Attention Metadata [24] and 


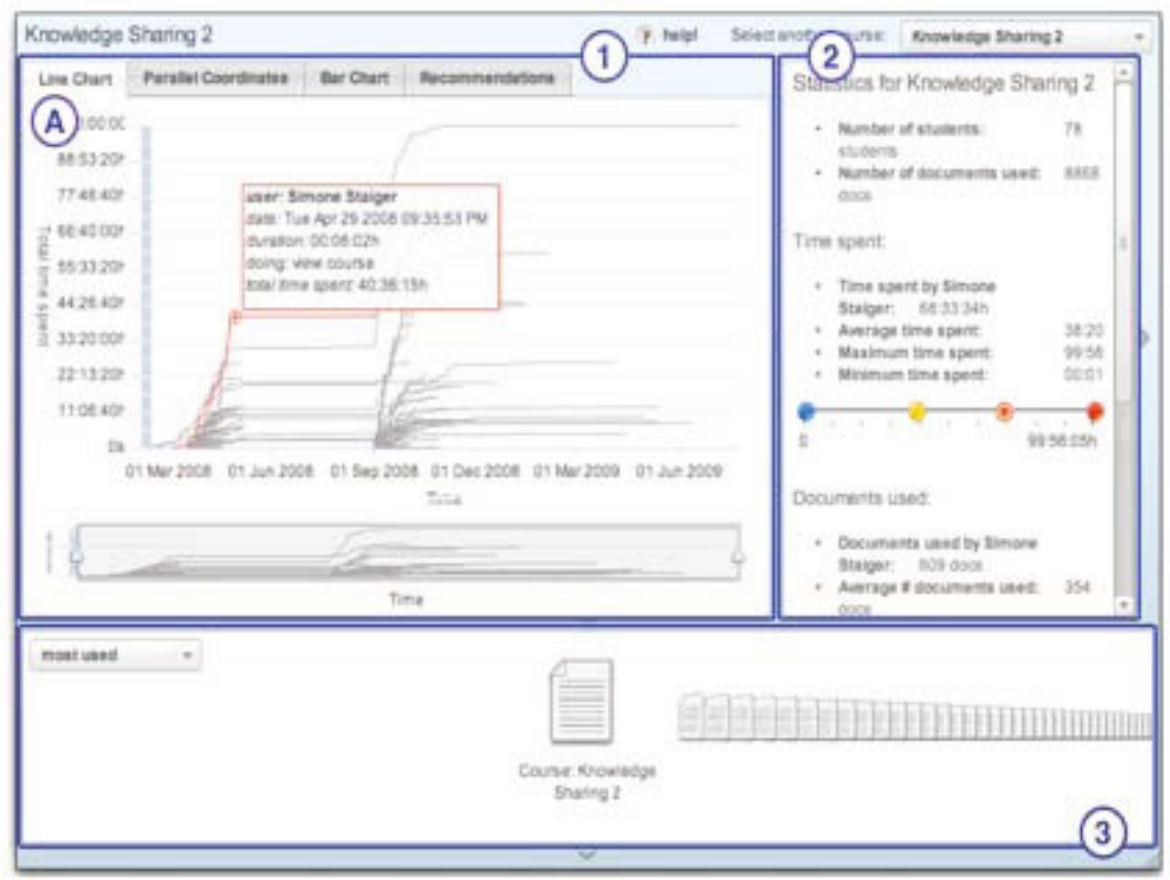

Fig. 7. The Student Activity Meter (SAM)

the UICO [17] models. Both models enable the capturing of user actions within an application, and potentially additional information about her current context, such as location or task related information.

The analysis of such data is a key enabler to gain insights into learning effects achieved and potential impact of technologies on learning. In addition, the visualization of such data has been researched extensively as a basis to support self-reflection, awareness and collaboration among learners or teachers [19]. One of our applications, SAM (Student Activity Meter), visualizes the time learners spent on learning activities [12]. Figure 7 shows some of the visualizations that SAM provides:

- The line chart (vis. A in figure 7) shows a line for every student, connecting all the timestamps when she was working with the cumulative amount of time spent. The inclination of the line shows the effort of the student. A steep line means an intensive working period. A flat line shows inactivity.

- Statistics of global time spent and document use are shown in box 2 in figure 7. Next to the actual numbers, a graphical view is presented with color coding of the minimum, maximum and average time spent and documents used, to give the user a visual comparison. Upon selection of a user in the visualization in box 1 , her statistics are also shown.

- The recommendation pane in box 3 allows to navigate through the most used and the most time spent on resources. 
Evaluation results indicate that such visualizations can be used successfully to increase awareness for teachers, i.e. of what and how students are doing. Resource usage can show teachers popular learning materials and enables resource discovery.

\section{Conclusion}

In this paper, we have argued that we are evolving towards an open learning infrastructure, much as originally envisioned in [14]. This evolution will make available an abundance of learning resources that will act as a motor of innovation. In this way, it will help to push further a research interest that permeates much of Hermann Maurers research.

Acknowledgments. Katrien Verbert is a Postdoctoral Fellow of the Research Foundation Flanders - FWO. The research leading to these results has received funding from the European Community Seventh Framework Programme (FP7/2007-2013) under grant agreement no 231396 (ROLE). The work presented in this paper is partially supported by the European Commission eContentplus programme - projects ASPECT (ECP-2007-EDU-417008) and ICOPER (ECP2007-EDU-417007). The research leading to these results has received funding from the European Community Seventh Framework Programme (FP7/2007-2013) under grant agreements no 231396 (ROLE) and no 257566 (iTEC).

\section{References}

1. 1st International Conference on Learning Analytics and Knowledge 2011 (2011)

2. Ariadne Foundation, http://www. ariadne-eu.org

3. GLOBE, http://globe-info.org/

4. LACLO: Latin-American Community of Learning Objects, http: //www. laclo.org/

5. IEEE 1484.12.1-2000 Standard for Learning Object Metadata (2000)

6. CEN Workshop Agreement CWA 15454 - A Simple Query Interface Specification for Learning Repositories (2005), ftp://ftp.cenorm.be/PUBLIC/CWAs/e-Europe/ WS-LT/CWA15454-00-2005-Nov . pdf

7. Anderson, C.: The Long Tail: Why the Future of Business Is Selling Less of More. Hyperion (2006)

8. Butoianu, V., Vidal, P., Verbert, K., Duval, E., Broisin, J.: User context and personalized learning: a federation of Contextualized Attention Metadata. Journal of Universal Computer Science (2010)

9. Dougiamas, M., Taylor, P.: Moodle: Using Learning Communities to Create an Open Source Course Management System. In: Lassner, D., McNaught, C. (eds.) Proceedings of World Conference on Educational Multimedia, Hypermedia and Telecommunications 2003, pp. 171-178. AACE, Honolulu (2003), http://www.editlib.org/p/13739

10. Duval, E., Forte, E., Cardinaels, K., Verhoeven, B., Durm, R.V., Hendrikx, K., Wentland-Forte, M., Ebel, N., Macowicz, M., Warkentyne, K., Haenni, F.: The ARIADNE knowledge pool system. Communications of the ACM 44(5), 72-78 (2001), http://portal. acm.org/citation. cfm?id=374308. 374346 
11. Fox, S., Karnawat, K., Mydland, M., Dumais, S., White, T.: Evaluating implicit measures to improve web search. ACM Trans. Inf. Syst. 23(2), 147-168 (2005), http://doi.acm.org/10.1145/1059981.1059982

12. Govaerts, S., Verbert, K., Klerkx, J., Duval, E.: Visualizing Activities for Selfreflection and Awareness. In: Luo, X., Spaniol, M., Wang, L., Li, Q., Nejdl, W., Zhang, W. (eds.) ICWL 2010. LNCS, vol. 6483, pp. 91-100. Springer, Heidelberg (2010)

13. Klerkx, J., Vandeputte, B., Parra Chico, G., Santos Odriozola, J.L., Van Assche, F., Duval, E.: How to share and reuse learning resources: the Ariadne experience. In: Wolpers, M., Kirschner, P.A., Scheffel, M., Lindstaedt, S., Dimitrova, V. (eds.) EC-TEL 2010. LNCS, vol. 6383, pp. 183-196. Springer, Heidelberg (2010), https://lirias.kuleuven. be/handle/123456789/280116

14. Marchionini, G., Maurer, H.: The roles of digital libraries in teaching and learning. Communications of the ACM 38(4) (1995), http://portal.acm.org/citation. cfm?id=205345

15. Morville, P.: Ambient Findability, vol. 11. O'Reilly Media, Inc., Sebastopol (2005), http://www . amazon.ca/exec/obidos/redirect?tag=citeulike09-20\\&amp; path $=$ ASIN $/ 0596007655$

16. Nagel, T., Pschetz, L., Stefaner, M., Halkia, M., Müller, B.: mæve - An Interactive Tabletop Installation for Exploring Background Information in Exhibitions. In: Jacko, J.A. (ed.) HCI International 2009. LNCS, vol. 5612, pp. 483-491. Springer, Heidelberg (2009), http://dx.doi.org/10.1007/978-3-642-02580-8_53

17. Rath, A.S., Devaurs, D., Lindstaedt, S.N.: UICO: an ontology-based user interaction context model for automatic task detection on the computer desktop. In: Proceedings of the 1st Workshop on Context, Information and Ontologies, CIAO 2009, pp. 8:1-8:10. ACM, New York (2009), http://doi.acm.org/10.1145/1552262.1552270

18. Schwartz, B.: The paradox of choice why more is less. HarperCollins e-books (2007), http://books . google. com/books?id=zutxr7rGc_QC\&printsec=frontcover

19. Soller, A., Martínez, A., Jermann, P., Muehlenbrock, M.: From Mirroring to Guiding: A Review of State of the Art Technology for Supporting Collaborative Learning. Int. J. Artif. Intell. Ed. 15(4), 261-290 (2005),

http://portal.acm.org/citation. cfm?id=1434935.1434937

20. Stefaner, M., Muller, B.: Elastic lists for facet browsers. In: Wagner, R., Revell, N., Pernul, G. (eds.) DEXA 2007. LNCS, vol. 4653, pp. 217-221. Springer, Heidelberg (2007)

21. Ternier, S., Massart, D., Assche, F.V., Smith, N., Simon, B., Duval, E.: A Simple Publishing Interface For Learning Object Repositories. In: Proceedings of World Conference on Educational Multimedia, Hypermedia and Telecommunications 2008, Chesapeake, VA, pp. 1840-1845 (2008), http://go.editlib.org/p/28625

22. Ternier, S., Verbert, K., Parra, G., Vandeputte, B., Klerkx, J., Duval, E., Ordonez, V., Ochoa, X.: The Ariadne Infrastructure for Managing and Storing Metadata. IEEE Internet Computing 13(4), 18-25 (2009), http://www . computer.org/portal/web/csdl/doi/10.1109/MIC. 2009.90

23. Van de Sompel, H., Nelson, M.L., Lagoze, C., Warner, S.: Resource Harvesting within the OAI-PMH Framework. D-Lib Magazine 10(12) (2004), http://www.dlib.org/dlib/december04/vandesompel/12vandesompel.html

24. Wolpers, M., Najjar, J., Verbert, K., Duval, E.: Tracking actual usage: the attention metadata approach. Educational Technology and Society 10(3), 106-121 (2007), https://lirias.kuleuven. be/handle/123456789/158649 